\title{
The Antibiotic Resistance Crisis - An Indian Perspective
}

\author{
Saini Devanshi and Dr. B. Lakshmi \\ Department of Pharmaceutical Management, NIPER Hyderabad, Hyderabad, Telangana, India \\ *Correspondence: Dr. B. Lakshmi, lakshmi.niperhyd@gov.in
}

\begin{abstract}
This research study reiterates the optimal usage of antimicrobial medicines in humans and animals to lessen antibiotic resistance. A primary survey was conducted to study an individual's role in prevention and control of antibiotic resistance. It was found that antibiotics are being irrationally used and the efficacy of antibiotics, as previously transforming the medical sciences and saving lives of many is in danger due to the quick emergence of bacterial resistance. Extensive efforts are therefore required to manage crisis by implementing new policies and renewing research efforts. Additionally, there exists a dire need to educate patients and public regarding antibiotic resistance crisis.
\end{abstract}

Keywords: Antibiotics, Antibiotic resistance crisis, Anti-microbial resistance (AMR), multidrug resistance (MDR), infection prevention and control (IPC)

\section{ARTICLE INFORMATION}

Author(s): Saini Devanshi and Dr. B. Lakshmi

Received: 06 Oct 2020; Accepted: 16 Nov, 2020; Published: 04 Dec 2020 ; e-ISSN: 2347-4696;

Paper Id: BMN-IJBMR-2020-12;

Citation: doi.org/10.37391/IJBMR.080404

\begin{tabular}{|l|l|}
\hline Cross \\
\hline CROSSREF
\end{tabular}

Webpage-link: https://ijbmr.forexjournal.co.in/archive/volume-8/ijbmr-080404.html

\section{INTRODUCTION}

WHO defines antibiotics as medicines used for preventing and treating bacterial infections. Alternatively, antibiotics can be described as chemical compounds that kill bacteria (cytotoxic)/slow their growth (cytostatic).

One chief approach of contemporary medicine which is used to combat infections is antibiotic treatment. The modern era of antibiotics ranged from the 1930s to 1960s. Several antibiotics like tetracycline, erythromycin etc. were discovered during this time. Unfortunately, this golden span ended because researchers were unable to maintain the pace of antibiotic discovery in the face of emerging resistant pathogens.

Antimicrobial resistance (AMR) is a serious concern to animal, human and environmental health. Emergence of MDR bacteria/superbugs can be ascertained to uncontrolled antibiotic access, gaps in IPC, inappropriate prescribing, extensive use in agriculture and insufficient new drug development by the pharma companies, ascribable to strict regulatory requirements and reduced finances.

\section{LITERATURE REVIEW}

Antibiotics are substances produced by microorganisms that selectively suppress growth of other microorganisms or kill them, at very low concentrations.

In 1928, Penicillin was the first discovered antibiotic. During WWII, it was used to combat bacterial infections among soldiers. Shortly then penicillin resistance became a considerable problem. In response, many new antibiotics (wonder drugs) were discovered and deployed [1]. Unfortunately, resistance has eventually been seen to nearly all magic bullets/wonder drugs developed in last 60 years [2].

\subsection{What is Antibiotic Resistance?}

It is a microorganism's ability to resist antibiotic consequences. Antibiotic resistance occurs when bacteria change in some way that reduces or eliminates the efficacy of drugs, chemicals, or other agents designed to cure or prevent infections. In these cases, the bacteria thrive, spreading and causing more damage.

\subsection{Causes of Antibiotic Resistance}

1. Misuse and Overuse: It is seen that people mistakenly take antibiotics to treat diseases such as virus caused influenza, when such drugs target and kill bacteria alone. Therefore, if anyone takes antibiotics for the wrong disease or uses too much too often, they kill helpful bacteria that populate body, threatening the delicate balance upon which their health depends. Furthermore, improper disposal of residual antibiotics eventually leads them to enter the food chain.

In addition, the bacteria are prone to mutation and evolution. So, when we take antibiotics at the wrong time or overuse them, resistant bacteria find it easy to replicate and spread, giving rise to additional strains of antibiotic resistant bacteria.

2. Inappropriate Prescribing: Some work also indicates that doctors often prescribe antibiotics erroneously or prescribe the wrong form of antibiotic, which has possibly led to the current health crisis. Incorrectly prescribed antibiotics have questionable therapeutic benefit and expose patients to potential complications of antibiotic therapy [3]. According to one study paper, $30-60 \%$ of antibiotics that doctors prescribe to people in ICUs are not necessary/inappropriate [4].

3. Substantial agricultural use: The rampant use of antibiotics in the food-animal production sector is another area of concern. Treating livestock with antimicrobials is said to 
improve the overall health of the animals, producing larger yields and a higher-quality product [5]. The antibiotics used in livestock are ingested by humans when they consume food, causing infections that may cause adverse health consequences [6].

The agricultural use of antibiotics also affects the environmental microbiome. Up to $90 \%$ of the antibiotics given to livestock are excreted in urine and stool, which is then dispersed through groundwater and surface runoff $[7,8]$.

A 2010 report estimating global antibiotic use in poultry, swine and cattle reveals that India accounts for 3\% of global consumption and is one of the world's top five users along with China, the US, Brazil and Germany. According to the same study, projections for 2030 estimate an $82 \%$ increase in antibiotic use for animal feed in our home country.

4. Availability of few new antibiotics: Economic and regulatory obstacles have stopped the pharmaceutical industry from producing new antibiotics, a strategy that had earlier been effective at combating resistant bacteria. The industry is rather focusing its efforts on developing profitable drugs used to treat chronic conditions that require lifelong daily treatment or on manifestations such as baldness or inadequate sexual performance [9].

A review of antibiotic patents confirms that, as for other drugs, pharmaceutical companies are still working more at modifying or combining existing antibacterial compounds than trying to find new chemical structures that could lead to new classes of antibacterial agents [10].

Meaning, these new compounds developed do not represent the true innovation, but are additions to existing classes of drug. The risk being that resistance to these new agents will emerge faster than for a drug with a truly new mechanism of action.

Additionally, microbiologists and infectious-disease specialists have advised restraint regarding antibiotic use.10 Therefore, once a new antibiotic is marketed, physicians - rather than prescribing it immediately_often hold this new agent in reserve for only the worst cases due to fear of promoting drug resistance, and they continue to prescribe older agents that have shown comparable efficacy $[6,11]$. As a result, new antibiotics are often treated as "last-line" drugs to combat serious illnesses. This practice leads to the reduced use of new antibiotics and a diminished return on investment [6, 11].

5. Regulatory barriers: For the companies that are optimistic about pursuing the discovery of new antibiotics, obtaining regulatory approval is often an obstacle $[11,12]$. Difficulties in pursuing regulatory approval that have been noted to include: bureaucracy, absence of clarity, differences in clinical trial requirements among countries, changes in regulatory and licensing rules, and ineffective channels of communication [12].

\section{METHODOLOGY}

A primary survey was conducted online in the month of April. The structured questionnaire was made on Google-forms and circulated online through social media platforms like WhatsApp, Facebook, LinkedIn, etc.

The sampling frame consisted of Indian citizens residing in multiple cities across the country and falling within the age bracket of 20 years or above. Of these, a sample size of 258 individuals, ones easily approachable while maintaining social distancing norms were chosen for the study. Majority of the questions were close ended, however a few of them were left open. Basic statistical tools and techniques were employed to analyse the collected data and graphs were obtained. Data involving various steps that can be taken at the level of policy makers, healthcare professionals, healthcare industry and agricultural sector was obtained through secondary research.

\section{RESULTS}

\subsection{Steps at Individual Level}

[1] Be aware: The study reveals that only 190 of all respondents know that antibiotics work against bacteria. 20 respondents believe they are effective against viruses, 2 individuals think of them to work against fungi, and 14 consider them to respond to all of the three - the bacteria, the fungi and the viruses. Another 29 individuals chose diverse combinations of options available. Lastly, 4 vague responses received include 2 indicating antibiotics to be effective against infection in general and other 2 believing them to work for cancer. It is of great importance to note that only 1 single respondent confessed of not knowing the correct answer.

[2] Use antibiotics only when prescribed by a certified health professional: It is seen that $60 \%$ individuals' resort to self-medication (antibiotics) for routine ailments like diarrhea, common cold, and influenza. What is to be paid attention is that the causative organisms behind all these ailments are not the bacteria but virus, against which antibiotics have no role to play.

[3] Gain spectrum knowledge: 34.9\% (90) respondents agree of knowing the spectrum of antibiotic they intake while $65.1 \%$ (168) respondents are unaware of the same.

[4] Be mindful of treatment regimen: It was observed that $79.5 \%$ individuals are mindful of taking an antibiotic until course completion and do not stop intake as and when they start feeling well.

[5] Never share or use leftover medicines: From the study results we understand that after course completion, a good majority of people (60.5\%) keep antibiotics in safe custody for the next time they fall sick. $32.2 \%$ of all individuals make a wise decision of disposing them off while still others give them away to friends and family members who complain of similar problems. 
[6] Make an attempt to gain information on healthcare challenges: One must understand that wealth is not restricted to money. Health is wealth too. Study shows that less than $50 \%$ individuals $(48.8 \%)$ understand the true meaning of antibiotic resistance - disease causing organism's resistance to antimicrobial that was once able to treat infection caused by that organism. $23.6 \%$ people on the other hand understand resistance as - a patient's/animal's resistance to antimicrobial drug used for the treatment of microbial disease, something which is incorrect.

[7] Make a sensible choice in terms of food habit and milk consumption: From literature it is understood that antibiotics are undoubtedly used for rearing livestock at poultry farms. As a result, on consumption of animal products/animals as whole, we tend to intake antibiotics indirectly. It is in this context that we should sensibly choose our food habit and milk consumption pattern until governmental rules get stringent with respect to antibiotic use at such places. Correlating an individual's food habit with how often he/she consumes milk, the following graph is arrived at.

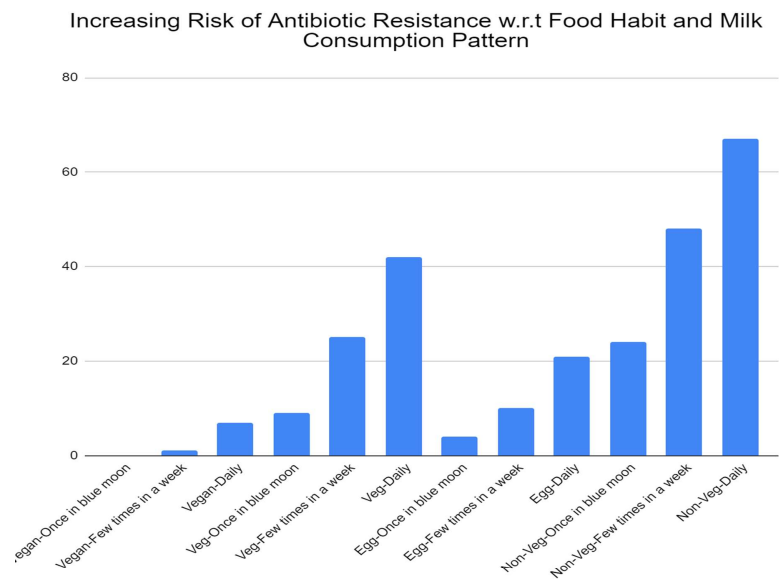

Figure 1: Risk of antibiotic resistance with respect to food habit and milk Consumption pattern

[8] Avoid infections by regularly washing hands, preparing food hygienically, avoiding close contact with sick people and practicing safer sex: It is quite delightful to state that $77.5 \%$ of all respondents wash their hands with water and soap always in contrast to washing hands with water alone. This indicates that a good number of people are doing their bit in preventing infections.

[9] Keep vaccinations up to date: It can be concluded that individuals are unsure of what vaccines they've undertaken. Such a bold statement can be made because DBT, BCG, Typhoid, Cholera and TT are mandatory in India. Even though people made random choices in answering this question, it is sure each one of my respondents residing in cities must have undertaken these in compliance with governmental norms.
[10] Be up to date with news and governmental measures: $77.5 \%$ of all respondents say that they are unaware of the Union Ministry of Health Affairs Red Line Campaign - a campaign that demands prescription-only antibiotics to be marked with a red line, to discourage their over-the-counter sale.

Also, when asked if people know in which month is the 'Antibiotic Awareness Week' celebrated, 152 people said they were not aware, 86 gave the correct answer of it being celebrated in the month of November and 20 people made wild attempts that turned out to be wrong.

\subsection{Steps for Policy Makers}

[1] To ensure that a comprehensive national action plan is in place to counter antibiotic resistance: The current NAP in India is extensive and well aligned with WHO's 'GAP for AMR'. It captures all five objectives as listed in the GAP and adds one extra objective related to strengthening India's leadership on AMR. The program aims to tackle many main aspects of AMR in both human and non-human sectors (such as agriculture, fisheries, animal husbandry, and environment) implementing the 'one health approach'. Although promising, the NAP has not yet come into full action in any of the Indian States so far.

[2] Improve surveillance of antibiotic-resistant infections: The national health authorities have recently recognized the value of AMR and only in 2017, the National Health Policy calls for a rapid standardization of guidelines on antibiotic usage, restriction of the usage of antibiotics as OTC medicines, prohibiting or limiting the use of antibiotics as animal growth promoters, and pharmacovigilance including prescription check inclusive of antibiotic usage - at hospital and in society.

[3] Strengthening policies, programs, and implementing infection prevention and control measures: Provisional infection management guidelines are posted on National Centre for Disease Control website as a template for hospitals to begin incorporating infection control strategies.

[4] Regulate and encourage the effective use and disposal of quality medicines: The Municipal Solid Waste Management (MSWM) Regulation, 2016 classifies expired and unused drugs as hazardous household waste. This waste is to be segregated and stored in separate bins (yellow in colour) and disposed in compliance with the Biomedical Waste Management Rules, 2016 that say the drugs should be incinerated at high temperatures.

[5] Make information available on the impact of antibiotic resistance: Despite various information, education and communication activities being conducted round the year (quiz competitions in schools, public lectures in academic institutions and radio programs, health fairs), the survey results show most people being unaware of the crisis or have misleading clue about the reasons of its existence. 


\subsection{Steps for Healthcare Professionals}

[1] Prescribe and administer antibiotics when appropriate, in accordance to current guidelines: As per WHO 2017 guidelines, antibiotics are divided in three distinct groups for which rules on how each class of drugs should be used to treat 21 of the most common infections are issued. Group I consist of medicines which should be made available to patients by prescription, for example - Amoxicillin. Group II comprises of carbapenems like Imipenem and Doripenem. Group III includes Colistin and other "last resort" antibiotics that must be seldom used, only for medical emergencies.

[2] Prevent infections by maintaining clean hands, tools/instruments, and environment.

[3] Report antibiotic-resistant infections to concerned authorities.

[4] Talk to patients about proper intake of antibiotics, antibiotic resistance and hazards of antibiotic misuse.

[5] Speak to patients about avoiding infections (for example, taking timely vaccinations, washing hands, safe sex, and nose and mouth protection while sneezing).

\subsection{Steps for Healthcare Industry}

[1] Invest in research and development of new antibiotics, vaccines, diagnostics and other tools: The DST, DBT, UGC and CSIR should foster R\&D in above mentioned domains. Also, new regulatory strategies are required to ensure that antibiotic medicines continue to evolve and become available, for example - Infectious Disease Society of America has proposed a new, limited-population antibiotic drug (LPAD) regulatory approval model which enables small, economic, and quick clinical trials.

\subsection{Steps for Agricultural Sector}

[1] Antibiotics should only be given to animals under veterinary supervision.

[2] Refrain from antibiotic usage for promoting growth/preventing diseases in healthy animals.

[3] Keep animal vaccinations up to date, reducing dependency on antibiotics.

[4] Use and encourage good practices at all stages of food production/processing.

[5] Improve farm biosecurity and hygiene to prevent animal infection.

\section{SUMMARY \& CONCLUSION}

Most individuals are aware of what antibiotics are yet $60 \%$ individuals take them for the treatment of viral infections.
Although people possess information regarding antibiotic course completion, they have little/no information at all regarding proper disposal of unused/leftover drugs, once the treatment course is complete.

Since we indirectly take antibiotics by consuming animal products/animals as whole, due consideration must be given to the food habit and milk consumption pattern we choose for ourselves until stringent laws are enforced to regulate antibiotic use in agriculture.

There exists a dire need to generate awareness among people about antibiotic resistance crisis and its impacts. Extensive promotion of the red line campaign along with spectacular celebrations of antibiotic resistance week can help in doing so.

Antibiotic-resistant infection surveillance should be scaled up. Continuous measures must also be undertaken for its improvement.

Doctors must strictly be asked to prescribe antibiotics in accordance to the regulatory guidelines.

There are a few laws regulating the use of antimicrobials in cattle, pigs and chicken raised for domestic consumption in India.

These regulations must be made strict and compulsory to abide by.

On international level, WHO has time and again taken measures to prevent/control antibiotic resistance along with generating awareness. Such efforts must be continued to tackle impending danger in time.

\section{REFERENCES}

[1] Ventola C. L. (2015). The antibiotic resistance crisis: part 1: causes and threats. P \& $\mathrm{T}$ : a peer-reviewed journal for formulary management, 40(4), 277-283.

[2] Zaman, S. B., Hussain, M. A., Nye, R., Mehta, V., Mamun, K. T., \& Hossain, N. (2017). A Review on Antibiotic Resistance: Alarm Bells are Ringing. Cureus, 9(6), e1403.

[3] Lushniak BD. Antibiotic resistance: a public health crisis. Public Health Rep. 2014;129(4):314-316.

[4] Luyt, C., Bréchot, N., Trouillet, J. et al. Antibiotic stewardship in the intensive care unit. Crit Care 18, 480 (2014). https://doi.org/10.1186/s13054-014-0480-6

[5] Michael CA, Dominey-Howes D, Labbate M. The antibiotic resistance crisis: causes, consequences, and management. Front Public Health. 2014; 2:145.

[6] Golkar Z, Bagazra O, Pace DG. Bacteriophage therapy: a potential solution for the antibiotic resistance crisis. J Infect Dev Ctries. 2014;8(2):129-136. 13.

[7] Centers for Disease Control and Prevention, Office of Infectious Disease Antibiotic resistance threats in the United States, 2013. Apr, 2013.

[8] Bartlett JG, Gilbert DN, Spellberg B. Seven ways to preserve the miracle of antibiotics. Clin Infect Dis. 2013;56(10):1445-1450. 
[9] P. Courvalin and J. Davies, Antimicrobials: time to act!, Curr. Opin. Microbiol. 6 (2003), 425-426.

[10] O.A. Phillips, Antibacterial agents: patent highlights January to June 2003, Curr. Opin. Investig. Drugs 4 (2003), 926-936.

[11] Gould IM, Bal AM. New antibiotic agents in the pipeline and how they can overcome microbial resistance. Virulence. 2013;4(2):185-191.
[12] Piddock LJ. The crisis of no new antibiotics - what is the way forward? Lancet Infect Dis. 2012;12(3):249-253.

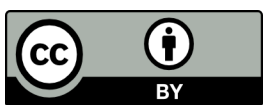

(C) 2020 by the Saini Devanshi and Dr. B. Lakshmi. Submitted for possible open access publication under the terms and conditions of the Creative Commons Attribution (CC BY) license (http://creativecommons.org/licenses/by/4.0/). 